\title{
Design and Evaluation of a Novel Floating Osmotic Pump System
}

\author{
Zhihong Zhang, Bo Peng, Xinggang Yang, Chao Wang, Guangmei Sun, Weisan Pan
}

Department of pharmaceutics, School of pharmaceutical science, Shenyang Pharmaceutical University, 103 Wenhua Road, 110016, Shenyang, People's republic of China.

Received, January 28 2009; Revised, April 18, 2009, Accepted, May 1, 2009; Published, May 1, 2009.

\begin{abstract}
Purpose. Find a novel delivery system for oral administration of drugs that have absorption window in the upper part of gastrointestinal (GI) track. Methods. Dipyridamole was chosen as the model drug. A novel system, which combined the osmotic pump controlled release system and the floating system was designed; matrix tablets (MT) were prepared for compares. The effects of $\mathrm{pH}$, temperature and hydrodynamic conditions on drug release and the floating behavior of floating osmotic pump system (FOP) was investigated. In vivo evaluation was performed by a three-crossover study in six Beagle dogs relative to the conventional tablet (CT). Cumulative percent input in vivo was compared with that of in vitro release profiles. Results. Floating behavior of FOP, drug releases from FOP and MT were sensitive to $\mathrm{pH}$ of dissolution media but not sensitive to temperature; the release of dipyridamole from MT was influenced by stirring rate while drug release from FOP was not. AUC of FOP was larger than MT and CT. The linear correlations between fraction absorbed in vivo and fraction dissolved in vitro was established for FOP-a true zero-order release formula, whereas only a nonlinear correlation was obtained for MT. Conclution. FOP could be a novel way for the oral administration for drugs like dipyridamole.
\end{abstract}

\section{INTRODUCTION}

Generally, hydroxy-propyl methylcellulose (HPMC) may provide many advantages as a hydrophilic matrix system with excellent process ability and many grades to choose from for formulation flexibility. However, the oral osmotic pump system has the advantage of in vivo predictability of release rate based on in vitro data. Therefore, there has been increasing interest in the development of oral osmotic pumps in the past 30 years. Various types of oral osmotic pumps have been developed and studied to deliver drugs possessing different aqueous solubility. In the 1970s, Theeuwes [1] developed the elementary osmotic pump (EOP) and brought forward its basic theory. However, EOPs are only suitable for moderately soluble drugs. Many effective ways have been found to increase drug solubility for improvement of drug-released rate. For example, convert drug into ionic substance by reacting with or adding alkali/acid [2, 3], using (SBE) ${ }_{7 \mathrm{~m}}-\beta-\mathrm{CD}$ as a solubilizer $[4,5]$. It is also acceptable for water-insoluble drug to be released in the form of suspension. Certainly, the 'push pull' osmotic pump (PPOP) is a case in point; many drugs were delivered via PPOP [6-9]. However, due to the complexity of the process of PPOP preparation, monolithic osmotic pump for water-insoluble drug has been employed. Liu et al. $[10]$ used polyethylene oxide (PEO) as a suspending and osmotic agent to prepare nifedipine monolithic osmotic tablet system (MOTS). Advantages and formulation aspects of various types of oral osmotic pumps were reviewed recently by Verma et al $[11,12]$. In addition, a large number of patent literature exists that describes novel osmotic systems [13].

The floating drug delivery system (FDDS), which can deliver drugs in the upper gastrointestinal (GI) tract, are considered for drug with an absorption window in the upper GI or for those that their solubility goes down as the media $\mathrm{pH}$ goes up. The concept of FDDS has been described in the literature as early as 1968 [14]. Based on the mechanism of buoyancy, two distinctly different technologies, i.e., effervescent and noneffervescent systems have been utilized in the development of FDDS. The effervescent systems utilize matrices prepared with swellable polymers such as methocel or polysaccharides, e.g., chitosan, and effervescent components,

\footnotetext{
Corresponding author: Dean, Prof. Dr. Weisan Pan, Department of pharmaceutics, School of pharmaceutical science, Shenyang Pharmaceutical University, 103 Wenhua Road, 110016, Shenyang, People's republic of China. E-mail: ppwwss@163.com
} 
e.g., sodium bicarbonate and citric or tartaric acid [15-17] or matrices containing chambers of liquid that gasify at body temperature [18]. When the preparations arrive in the stomach, carbon dioxide is liberated by the acidity of the gastric contents and is entrapped in the gellified hydrocolloid. This produces an upward motion of the dosage form and maintains its buoyancy. The most commonly used excipients in noneffervescent FDDS are gel-forming or highly swellable cellulose type hydrocolloids, polysaccharides, and matrix forming polymers such as polycarbonate, polyacrylate, polymethacrylate and polystyrene. Ali et al [19] published have discussed a hydrodynamically balanced system (HBS) capsule containing a mixture of a drug and hydrocolloids. Upon contact with gastric fluid, the capsule shell dissolves; the mixture swells and forms a gelatinous barrier thereby remaining buoyant in the gastric juice for an extended period. Xu and Sun [20] have prepared matrix-floating tablets that were hydrodynamically balanced in the stomach for an extended period. Hydroxypropyl methylcellulose K4M and Carbopol 971P NF were used in the system. Harrigan [21] have developed a floating system, known as an intragastric floating drug delivery device. The device comprised of a drug reservoir encapsulated in microporous compartment with pores along its top and bottom surfaces. The peripheral walls of the drug reservoir compartment were completely sealed to prevent any physical contact of the undissolved drug with the stomach walls. The floatation chamber caused the system to float in the gastric fluid. Much more about floating systems has been reviewed by Singh and Kim [22].

A system that combined the osmotic pump controlled release system and the floating system was designed in this research. The system consisted of an MOTS and an aerocyst (part of the capsule filled with air) to provided the buoyancy (Figure1). The MOTS was locked inside the capsule using an enterosoluble capsule cap with a friction to prevent the MOTS from dropping form the capsule cap. A tiny aperture with a diameter about $0.6 \mathrm{~mm}$ was drilled on its top of capsule cap before the two parts joined. The purpose of the aperture was to avoid the disintegration caused by the high air pressure, see Figure1 part B.

Dipyridamole is a platelet inhibitor that is primarily recognized as an antithrombotic agent. Binding of adenosine with the adenosine receptor stimulates adenylate cyclase activity and production of cyclic AMP (cAMP). Elevated cAMP impairs platelet aggregation and causes arteriolar smooth muscle relaxation [23].

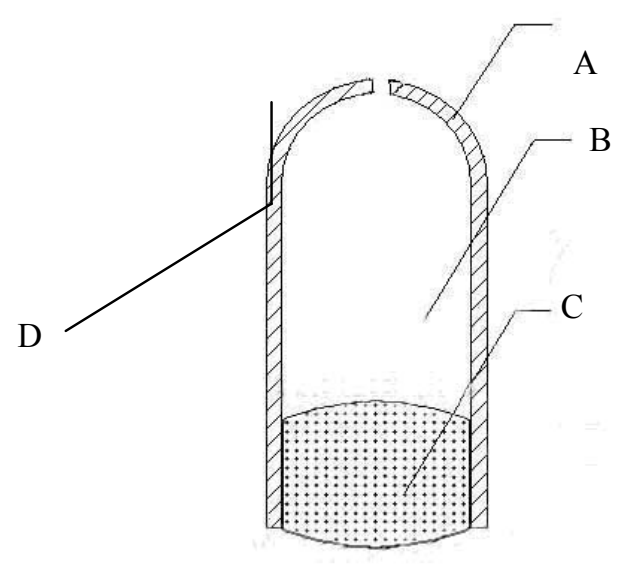

Figure 1. The structure of the FOP. Key: A: capsule cap (with an aperture on the top); B: air; C: MOTS; D: the aperture.

Because of its short biological half-life of 2-3 h, it should be frequently administered. In the recent statement from the American Heart Association and American Stroke Association Council on Stroke, extended release dipyridamole is considered safe. The solubility of dipyridamole is rather high in acidic solution, but poor in neutral or alky media [24]. Dipyridamole may be absorbed mainly in the first part of the GI tract; hence, it would be a suitable candidate for a floating dosage form.

It is realized that the in vivo bioavailability study of floating preparation should be carried out in human $[25,26]$ or in rabbits $[19$, 27] as a suitable animal model due to the similarity in the position of the stomach. The physical position of the stomach in dogs and human is also similar when they keep standing. Therefore, it is acceptable to carry out in vivo study in dogs. It has been found that osmotic tablets are not affected by food intake and in vitro stirring rate as much as matrix tablets [28].

\begin{tabular}{|l|}
\hline Abbreviations: \\
GI: gastrointestinal \\
MT: matrix tablets \\
FOP: floating osmotic pump system \\
CT: conventional tablet \\
EOP: elementary osmotic pump \\
PPOP: push pull osmotic pump \\
PEO: polyethylene oxide \\
MOTS: monolithic osmotic tablet system \\
FDDS: floating drug delivery system \\
Xti and Xti: average cumulative release \% of two \\
different formulations on point $i$ \\
CV: coefficient of variation \\
LOQ: limit of quantitation \\
CA: cellulose acetate
\end{tabular}


In this paper, two distinct controlled release preparations of dipyridamole, a floating osmotic pump and a hydrophilic matrix tablet were prepared and tested in formulating. The in vitro drug dissolution, in vivo drug absorption and bioavailability of two formulations were evaluated. Moreover, in vitro dissolution and in vivo absorption correlations were investigated.

\section{MATERIALS AND METHODS}

\section{Materials}

Dipyridamole (>99\%) was presented from Shenyang NO.1 Pharmaceutical Factory (Shenyang, China). HPMC (K4M, K15M, and K100M) were obtained from Shanghai Colorcon Coating Technology Limited for free (Shanghai, China). Silicon dioxide was purchased from Guangzhou Renmin Chemical Plant (Guangzhou, China). Polyoxyethylene (PEO WSR303) was given by Dow Chem. Co. (New Jersey, USA). Sodium chloride $(\mathrm{NaCl})$ and glucose were purchased from Shenyang Chemical Factory (Shenyang, China). Cellulose acetate (CA, 54.5-56.0 wt.\% acetyl content) was purchased from Shanghai Chemical (Shanghai, China). Polyethylene glycol (PEG) was purchased from Pudong Gaonan Chemical (Shanghai, China). Magnesium stearate (M.S.) was purchased from Shanghai Pharmaceutical
Excipients Factory (Shanghai, China). Hydrochloric acid $(\mathrm{HCl})$ was purchased from Shenyang Chemical Reagent Company (Shenyang, China). Methanol was purchased from Jiangsu Hanbon Sci \& Tech. Co. Ltd., Jiangsu, China. Glacial acetic acid (HAc) and triethylamine (TEA) was purchased from Tianjin Bo-di Chemical Industry, Tianjin, China. All reagents used were of either HPLC or analytical grade.

Granules of the MOTS were formulated by dry granulation method. The ingredients (listed in Table 1) were mixed well; the mixed powder was compressed into pieces; then the pieces were shattered and passed through a mesh $(1150 \mu \mathrm{m})$. Subsequently, the granules were lubricated with magnesium stearate and compressed by a single station- punching machine with concave punches. The average hardness of compressed tablets was found to be $10.0 \pm 0.5 \mathrm{~kg} / \mathrm{cm} 2$. Cellulose acetate (CA, 3\%, w/v) containing known levels of pore former was used as a coating solution, and core tablets were spray-coated. After coating, the tablets were dried at $40^{\circ} \mathrm{C}$ for $12 \mathrm{~h}$. An exit port was mechanically drilled through the membrane with a $0.6 \mathrm{~mm}$ drill on each side of the tablets. A same size tiny aperture was mechanically drilled through the capsule caps on the top. Finally, the MOTS was attached to the caps to form FOP.

\section{Preparation of FOP and MT}

Formulations were prepared according to different formulation principles (Table 1).

Table 1: Composition of two controlled release preparations.

\begin{tabular}{ll} 
MOTS of FOP & \\
\hline Ingredients & \\
Tablet core & Excipient / tablet core, w/w (\%) \\
Dipyridamole & 24.8 \\
WSR303 & 14.9 \\
NaCl & 24.8 \\
Glucose & 34.7 \\
Magnesium stearate & 1.0 \\
Coating & \\
PEG/coating, w/w (\%) $)$ & 50.0 \\
Coating gain up (\%) & 8.5 \\
Matrix tablets & \\
Ingredients & Excipient / tablet core, w/w (\%) \\
Dipyridamole & 25 \\
HPMC K4M & 28.75 \\
NaCl & 38.75 \\
Silicon dioxide & 7.5 \\
\hline
\end{tabular}


Dry granulation technique was applied to the matrix sustained release tablets preparation. Dipyridamole HPMC K4M and $\mathrm{NaCl}$ were mixed uniformly. The mixture was compressed into pieces; then the pieces were shattered and passed through a mesh $(1150 \mu \mathrm{m})$. After that, the granules were mixed with Silicon dioxide and compressed by a single station- punching machine with concave punches.

\section{In vitro drug release test}

The in vitro release test was carried out by using $900 \mathrm{ml}$ of $0.1 \mathrm{~N} \mathrm{HCl}$ as the medium in USP paddle apparatus at $37 \pm 0.5^{\circ} \mathrm{C}$ and $50 \mathrm{rpm} .5 \mathrm{ml}$ of samples were taken at $1,2,4,6,8,10$ and $12 \mathrm{~h}$, and filtered through a $0.8 \mu \mathrm{m}$ filter membrane. $5 \mathrm{ml}$ of fresh dissolution medium was added after each sampling.

The content of dipyridamole was determined at 282 $\mathrm{nm}$ by UV spectrophotometry. The mean calibration curve of dipyridamole $(\mathrm{C}=19.258 \mathrm{~A}$ $0.3601)$ was linear between 0.99 and $11.90 \mu \mathrm{g} / \mathrm{ml}$.The correlation coefficient of the calibration curve was 0.9999 .

The profiles were compared using similarity factor (f2) given by SUPAC guidelines for modified release dosage form, the equation is as follow [29]:

$$
f 2=50 \lg \left\{\left[1+1 / \mathrm{T} \sum(\mathrm{Xti}-\mathrm{Xri})^{2}\right]^{-1 / 2} \times 100\right\}
$$

Where, Xti and Xti are the average cumulative release $\%$ of two different formulations on point $i$, respectively; $T$ is the sample quantity in the dissolution test per preparation with the following restrictions: The dissolution profiles are considered to be similar when $f 2$ is between 50 and 100 . The dissolution measurements of the test and reference batches must be made under exactly the same condition. There should only be one measurement considered after either product has achieved 85\% dissolution. The percent coefficient of variation at the earliest point should not exceed $20 \%$ and the CV (\%) should not exceed 10 at all other time points.

\section{In vivo bioavailability study}

A three-crossover design in Beagle dogs was employed to compare the difference between the FOP, MT and the conventional dipyridamole tablet in vivo. The dose schedule was decided according to the characteristic of different dosage form. Based on instruction, conventional dipyridamole tablets should be administrated three times a day, 25-50 mg per time for an adult; sustained release capsules should be administrated two times a day, $200 \mathrm{mg}$ per time for an adult. On the consideration of the limit of quantitation (LOQ) of dipyridamole and the possibility of the bioavailability enhancement of FOP, the administration dosages were as follows: CT $150 \mathrm{mg}$ per dog; MT $400 \mathrm{mg}$ per dog; FOP 200 $\mathrm{mg}$ per dog. Six Beagle dogs were open-labeled and fasting $12 \mathrm{~h} .5 \mathrm{ml}$ of blood samples were collected at predetermined time intervals after administration. Samples were immediately centrifuged at $4000 \mathrm{rpm}$ for $10 \mathrm{~min}$. The plasma was separated and frozen at $-20^{\circ} \mathrm{C}$ before analysis. Analytical method of dipyridamole in plasma was developed according to literatures $[30,31]$. Glipizide was taken as internal standard, plasma sample was extracted twice using acetoacetate. Then the acetoacetate sample was evaporated to dryness, and the dried samples were reconstituted in methanol. Finally, the solution was injected into the high performance liquid chromatograph (HPLC). The HPLC condition: Diamonsil $\mathrm{C}_{18}(250 \mathrm{~mm} \times 4.6 \mathrm{~mm}, 5$ $\mu \mathrm{m})$; and mobile phase which consisted of methanol/water/HAc/TEA $\quad(70: 30: 0.3: 0.1, \quad \mathrm{v} / \mathrm{v} / \mathrm{v} / \mathrm{v})$, pumped at a flow rate of $1.0 \mathrm{~mL} \cdot \mathrm{min}^{-1}$. Concentrations of $0.05-20.0 \mu \mathrm{g} \cdot \mathrm{mL}^{-1}$ were linear and the minimum quantifiable concentration was $20 \mathrm{ng} \cdot \mathrm{mL}^{-1}$.

\section{RESULTS}

\section{Preparation design}

The thickness of the membrane determined the combination of the MOTS and the capsule caps; the combination was optimal when the coating weight gain was between $7-10 \%, 8-9 \%$. Dipyridamole release was complete and all the FOP floated over 12 hours while sank after 24 hours in dissolution test.

The orifice size did not have much influence on the drug release rate. It was also found that the orifice size did not influence the drug release profiles in the range of 0.25 to $1.41 \mathrm{~mm}$ [10]. The FOP floated well over 12 hours when the size of tiny aperture on capsule caps was $0.4 \mathrm{~mm}$ and $0.6 \mathrm{~mm}$. However, when the aperture on capsule caps was $0.9 \mathrm{~mm}$, the FOP sank in 10 hours. $0.6 \mathrm{~mm}$ orifice was chosen on the consideration that when the orifice size was $0.4 \mathrm{~mm}$, the orifice might be jammed by the drug or excipients [32].The osmotic system had the potential to deliver drug in a zero-order fashion between 0 and $10 \mathrm{~h}$ as long as orifice diameter had been chosen. In the narrow scope of coating gain up of $8-9 \%$, the release profiles had no significant difference (f2 was 
$66.5>50)$, the release rates were mainly regulated by the amount of PEG using in the coating (Figure2 and Figure3). On the consideration of the time how long people could keep out of lie down, a formulation with $10-12 \mathrm{~h}$ control release was expected. Though the release behavior was similar between the formulation using 50\% and $40 \%$ PEG in coating, the cumulative release of the formulation using 50\% PEG in coating was much higher at the end, so it was chosen as an optimal formulation. Dipyridamole release from FOP was complete and all the FOP floated over 12 hours, while sank after 24 hours in dissolution test.

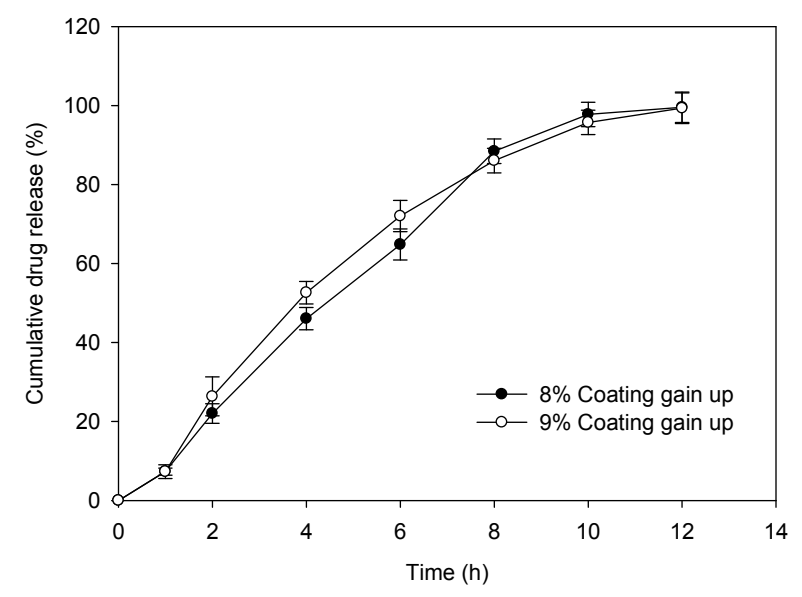

Figure 2. Influence of membrane thickness on dipyridamole release behavior $(n=6)$.

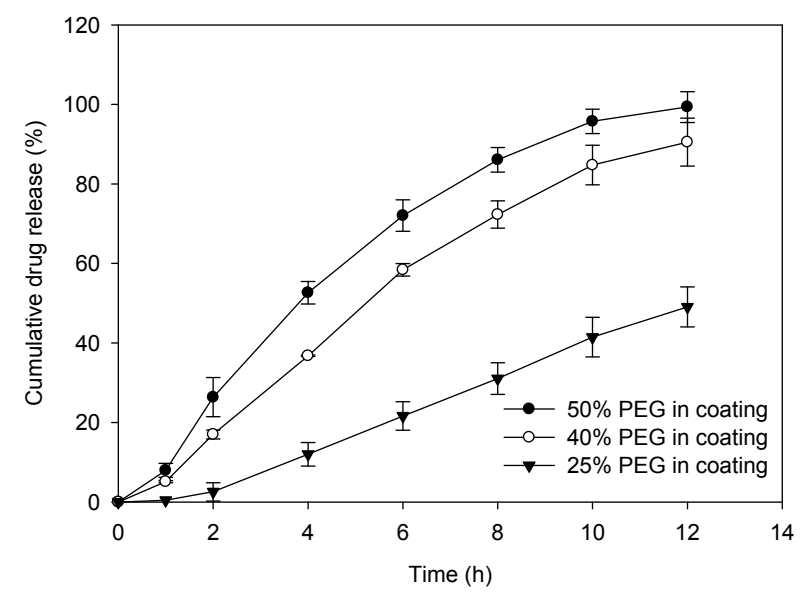

Figure 3. Influence of PEG amount on dipyridamole release behavior $(n=6)$.

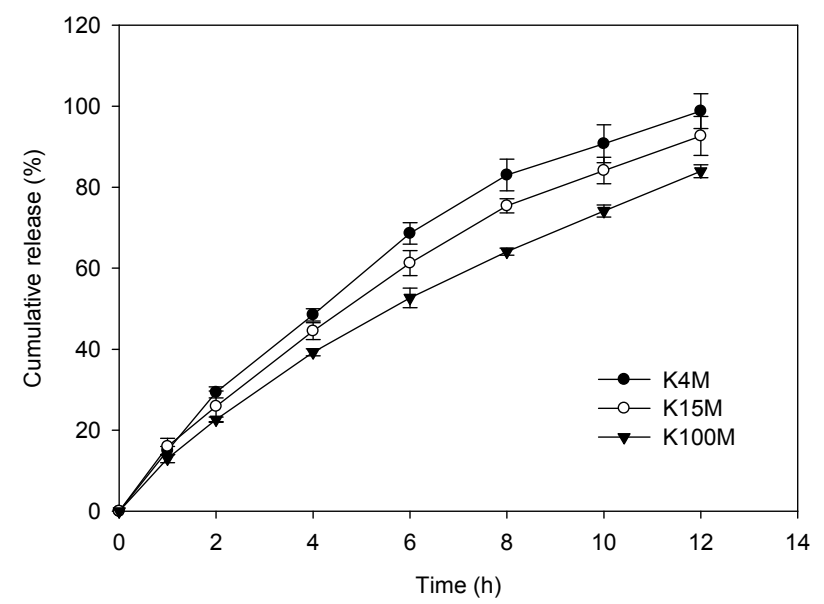

Figure 4. Effect of the viscosity of HPMC on dipyridamole release behavior $(n=6)$.

For matrix tablet, micronization silicon dioxide was employed because dipyridamole has very poor fluidity and silicon dioxide amount used in oral solid dosage form could be much larger than magnesium stearate, FDA suggests silicon dioxide used in sustained release tablets could get to the maximum of $170 \mathrm{mg} / \mathrm{d}$. From Figure 4 it could be seen that the viscosity of HPMC has some influence on dipyridamole release because the low solubility of dipyridamole. The higher viscosity of polymer was used, the slower rate of polymer hydration/erosion was produced. The $\mathrm{f} 2$ between $\mathrm{K} 4 \mathrm{~m}$ and $\mathrm{K} 100 \mathrm{M}$ was $43.6<50$.

\section{Evaluation of formulation}

Dipyridamole dissolution test should be done under the condition of $0.1 \mathrm{~N} \mathrm{HCl} 900 \mathrm{ml}$ as medium, paddle apparatus at $37^{\circ} \mathrm{C}$ and $50 \mathrm{rpm}$. In such condition, the profiles of drug release from FOP and MT were similar (Figure5), and $\mathrm{f} 2$ factor was 63.6.

To study the effect of temperature on the drug release profiles, dissolution tests of the optimal formulation (shown in table 1) was carried out at $36^{\circ} \mathrm{C}, 37^{\circ} \mathrm{C}$ and $39^{\circ} \mathrm{C}$. It was found that an increase in temperature did not significantly affect the release rate of drug from FOP or MT, floating time of FOP either.

To study the effect of stirring rate on the drug release profiles, dissolution tests of the optimal formulations were carried out at stirring rates of 25 , 50 and $100 \mathrm{rpm}$. It was shown that an increase in the rate of stirring did not significantly affect the release rate of drug from FOP, and the floating time 
of FOP were over 12 hours. Thus, the mobility of the gastrointestinal tract might hardly affect the drug release of the FOP. However, when the effect of hydrodynamics was evaluated using MT, it was found that the release of dipyridamole was significantly faster with the increase of the stirring rate (Figure 6). Potentially, matrix tablets might be more variable when tested in vivo, compared to FOP that is later confirmed by the in vivo study.

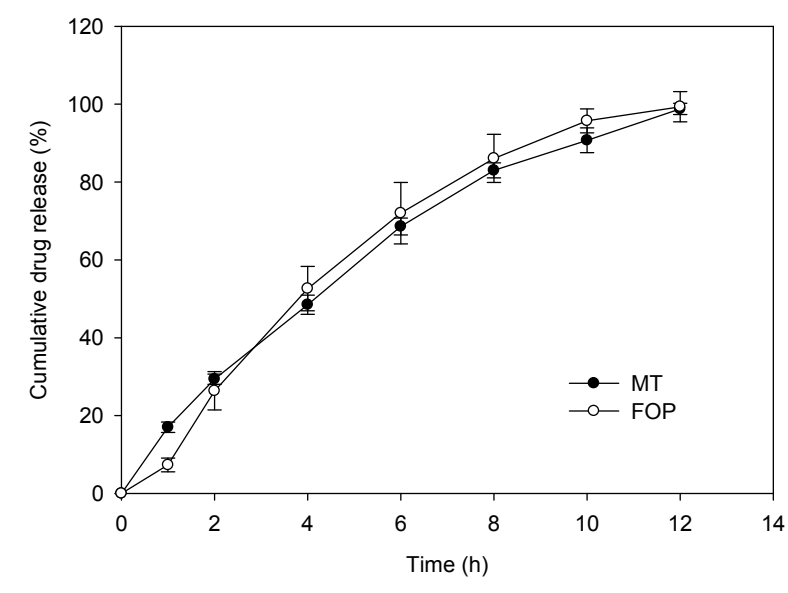

Figure 5. In vitro dissolution profiles of dipyridamole release from FOP and MT $(n=6)$.

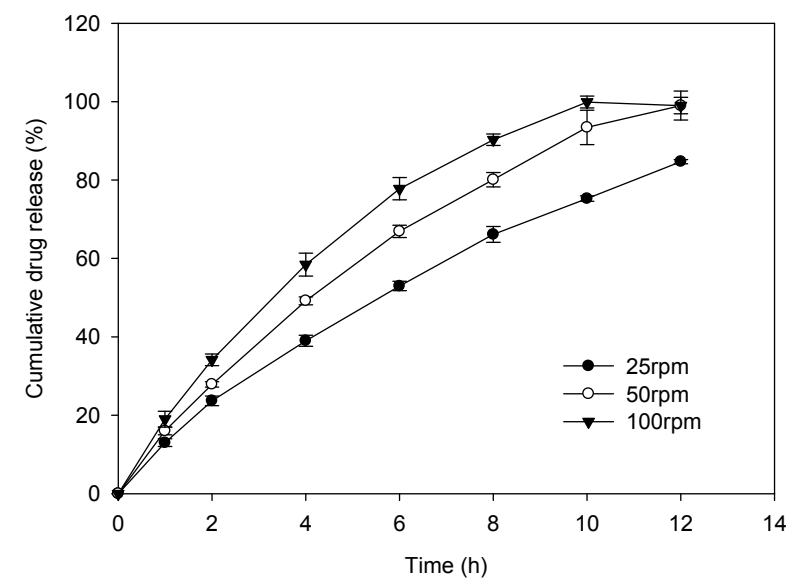

Figure 6. Effect of the stirring rate on dipyridamole release from MT $(n=6)$.

In order to simulate the physiological environment in human, the in vitro release tests of the optimal formulations were conducted in $0.1 \mathrm{~N} \mathrm{HCI}$ for the first $2 \mathrm{hr}$ and changed to $\mathrm{pH}$ 6.8-phosphate buffer for the following $10 \mathrm{hr}$. The release profiles of FOP and MT were similar; both were affected by the media $\mathrm{pH}$. The release rate of both FOP and MT slowed down in the $\mathrm{pH} 6.8$ phosphate buffer (Figure 7).

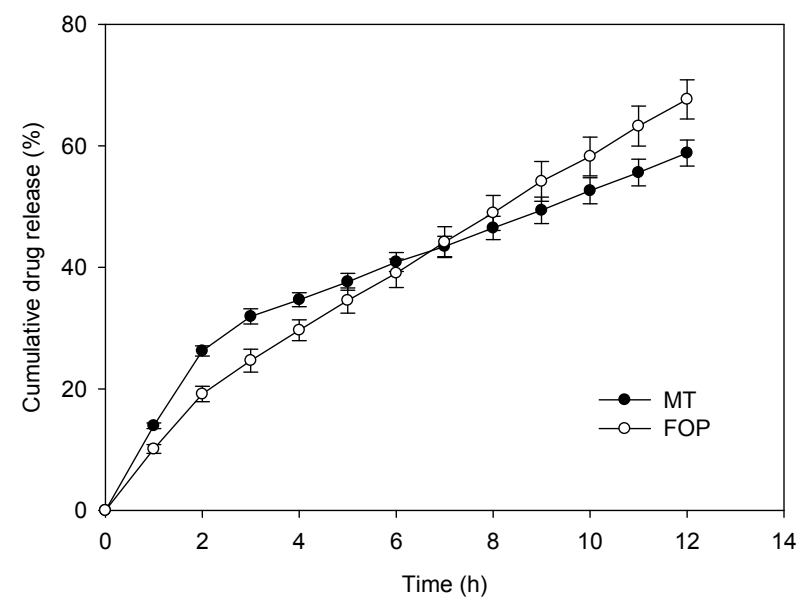

Figure 7. In vitro Release of dipyridamole from FOP and MT, using 0.1N HCI (0-2 hr) and pH 6.8 phosphate buffer $(2-12 \mathrm{hr})$ as medium $(\mathrm{n}=6)$.

\section{In vivo test and in vitro in vivo correlation evaluation}

Concentrations of dipyridamole in blood after oral administration of two modified released preparations were shown in Figure8. Measurable levels of dipyridamole were present at the last sample time point, $24 \mathrm{~h}$ post doses.

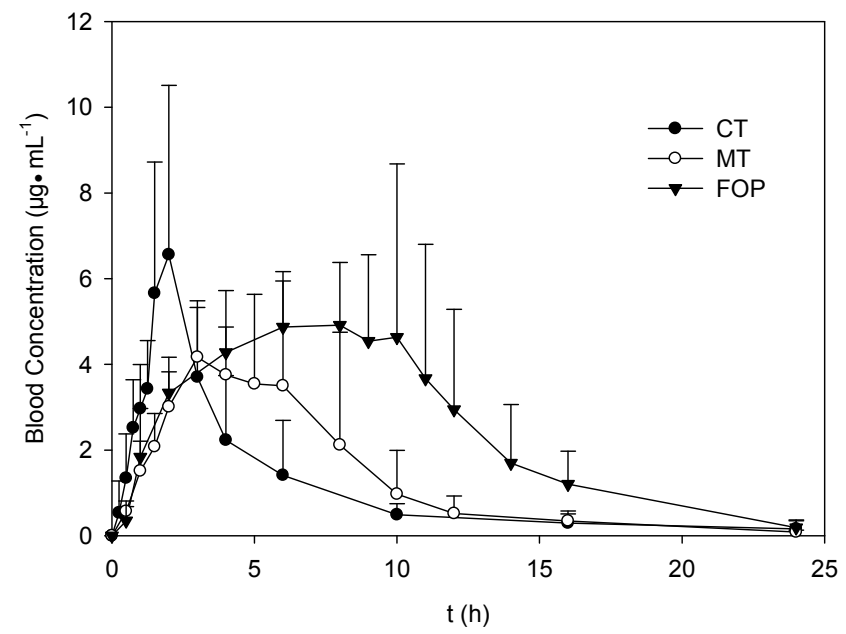

Figure 8. The time course of dipyridamole in Beagle dogs following administration of a single oral dose as $150 \mathrm{mg}$ (CT), 400 (MT) and 200 (FOP) ( $\mathrm{n}=6$ / group). 
Firstly, it could be seen that both the FOP and MT generated at a nearly constant blood level of dipyridamole. This could be a preliminary indication that true zero-order release was obtained for both FOP and MT under the in vivo environment. However, the period of the sustained plasma level of EOP was much longer than that of MT obviously.

Then the areas under the plasma concentration time curve (AUC) were calculated using statistical moment theory [33] and compared. The AUC of dipyridamole of the MT yielded to a relative oral bioavailability of $119.4 \%$ to CT. In addition, the AUC of dipyridamole of the FOP yielded to a relative oral bioavailability of $213.7 \%$ to $\mathrm{CT}$ and $179.0 \%$ to MT, respectively. It could be considered that the MT is bioequivalent to CT, while FOP had a higher bioavailability than either CT or MT. In other words, FOP enhanced the bioavailability of dipyridamole. However, on the consideration of the dosage administrated in the in vivo test, the dosage of FOP per unit should be regulated to be bioequivalent to the commercial available dosage forms in the future study.

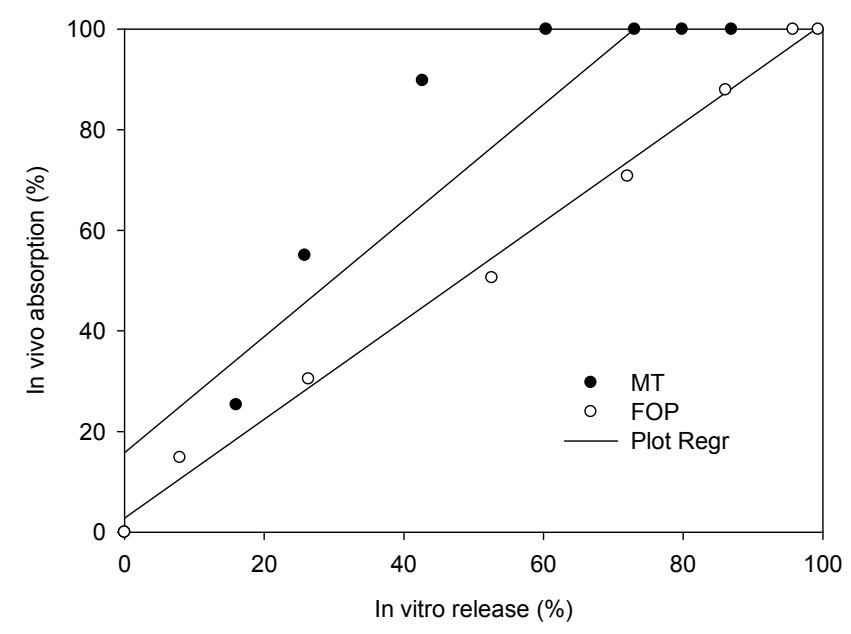

Figure 9. IVIVC model linear regression plots of $\%$ absorbed vs. $\%$ dissolved.

Percent in vivo input (\%dose) was plotted as a function of time and compared with percent dissolved in vitro to seek an in vivo-in vitro correlation. For FOP, the absorption was close to zero order with a slight decrease after $10 \mathrm{~h}$. When $\%$ in vivo input was plotted vs. \% dissolved (Figure9), the linear correlations were obtained, \% in vivo input $=0.9584 \%$ dissolved +4.6892 , $\mathrm{r}=0.9964$. It indicated that a close correlation could be established between the in vitro releases of FOP with its in vivo absorption. Although there was only one formulation for the osmotic system, an IVIVC was very meaningful in the identification of the floating property in vivo since the release from the FOP was independent of in vitro test conditions except media $\mathrm{pH}$.

For MT, a correlation could not be established by comparison of the in vivo input (\%dose) profile to the in vitro release profile (Figure9). The curvature of fraction absorbed in vivo vs. fraction dissolved in vitro for hydrophilic matrix tablets indicated that there was a time-scale difference between in vivo and in vitro testing. In vitro dissolution did not over $85 \%$ until $12 \mathrm{~h}$, while in vivo absorption input achieved $100 \%$ in $6 \mathrm{~h}$ after administration. It could be presumed that the dipyridamole might be absorbed in the upper part of GI, in 6 hours after administration the MT was in the upper part of GI, after that the drug was hardly absorbed. This could explain why the bioavailability of MT was much lower than FOP even though the administration dosage was larger than FOP in the in vivo tests was.

Taken together, it can be seen that in vitro-in vivo correlation is very important to the extended release oral dosage forms.

\section{DISCUSSION}

Dipyridamole was formulated into controlled release formulations exhibiting comparable in vitro release profiles using two formulation principles, i.e. floating osmotic pump system and hydrophilic matrix tablet. From the in vitro dissolution test, we could come up with the conclusion that the mobility of the gastrointestinal tract might hardly affect the drug release from the FOP while affect the drug release from the MT much. The bioavailability was elevated when the drug was made into FOP compared to MT. Drug release from the FOP was independent of in vitro and in vivo conditions, where best sustained release effect was achieved, whereas the in vitro dissolution test employed for hydrophilic matrix tablets needed to be optimized to be biorelevent. It could be considered that the floating osmotic system was a good vehicle for delivery of dipyridamole.

Dipyridamole is a water insoluble molecule. It was a great challenge for the pharmacists to design its formulation, especially to design the MOTS. In this study, we found that the solubility of dipyridamole increased in the lower $\mathrm{pH}$ media. So citric acid was used to modulate the solubility of dipyridamole within the core. Nevertheless, the 
powder mixture contained acid absorbed moisture so much and so fast that it could not be made into granule or compressed into tablets. The way to overcome the difficulty was to utilize PEO WSR303 as a suspending agent, swelling agent and osmotic agent to prepare the tablet formulation. Cellulose acetate (CA) was used as semi permeable membrane and polyethylene glycol 4000 (PEG-4000) was employed as plasticizer and pore former for controlling membrane porosity.

Many factors that might influence the drug release behavior of osmotic pump have been reported in literatures [11, 32], in this paper, the influence of coating level and orifice size on the drug release was studied.

Mechanism for FOP, when the system came in contact with the aqueous environment of the GI tract, the aerosol provided buoyancy to keep the system floating on the top of the aqueous. The osmotic core imbibed water from the surrounding medium via the semi permeable membrane and formed a suspension of dipyridamole inside the device. Sodium chloride provided a great osmotic pressure and imbibed water persistently, and PEO swelled with water. As the membrane was not extensible, the suspension of dipyridamole was pushed out though the orifice because of the high pressure caused by the increase in volume of the tablet core. The pressure was relieved by the flow of suspension out of the device through the delivery. This process continued at a constant rate until the entire solid agent inside the tablet had been hydrated; then the hydrated core continued to be delivered at a declined rate until the difference of pressure between inside and outside the membrane was disappeared. At the same time, water went into the aerosol though the tiny hole on the top of capsule cap slowly. After a long time, the aerosol contained certain water and the density of the system was close to the surrounding media. Then FOP suspended or sank in the GI aqueous, and went down though the GI tract along with the aqueous.

For MT, it could be seen that the in vitro release data of dipyridamole was found to follow zero kinetics $(\mathrm{r}=0.9803)$. Poorly water-soluble drug was released predominately by the erosion mechanism.

\section{REFERENCES}

[1]. Theeuwes, F. Elementary osmotic pump. J Pharm Sci, 1975; 64:1987-1991.

[2]. Lu EX, Jiang ZQ, Zhang QZ, Jiang XG. Preparation of controlled release coated tablets of naproxen sodium. Chin Pharm J, 2002; 37:841-844.

[3]. Defang O, Shufang N, Wei L, Hong G, Hui L, Weisan Pan. In vitro and in vivo evaluation of two extended release preparations of combination metformin and glipizide. Drug Dev Ind Pharm, 2005; 31:677-685.

[4]. Okimoto K, Rajewski RA, Stella VJ. Release of testosterone from an osmotic pump tablet utilizing $(\mathrm{SBE})_{7 \mathrm{~m}}-\beta-\mathrm{CD}$ as both a solubilizing and an osmotic pump agent. J Control Release, 1999; 58:29-38.

[5]. Okimoto K, Tokunaga $\mathrm{Y}$, Ibuki R, lrie $\mathrm{T}$, Uekama K, Rajewski RA, Stella VJ. Applicability of (SBE) $7 \mathrm{~m}-\beta-\mathrm{CD}$ in controlled-porosity osmotic pump tablets (OPTs). Int J Pharm, 2004; 286:81-88.

[6]. Swanson DR, Barclay BL, Wong PSL, Theeuwes F. Nifedipine gastrointestinal therapeutic system. Am J Med, 1987; 83:3-9.

[7]. Kuczynski AL, Ayer AD, Patrick SLW. Oral hypoglycemic glipizide granulation.US Patent: 5024843, Jun 18, 1991.

[8]. Gan Y, Pan W, Wei M, Zhang R. Cyclodextrin Complex Osmotic Tablet for Glipizide Delivery. Drug Dev \& Ind Pharm, 2002; 28:1015-1021.

[9]. Wu X, NIE S, Zhang N, Zheng Q, Hou P, Pan W. Preparation and in vitro release of nisoldipine two-layer osmotic pump tablets. Chin Pharm J, 2004; 39:46-48.

[10]. Liu L, Khang G, Rhee JM, Lee HB. Monolithic osmotic tablet system for nifedipine delivery. J Control Release, 2000; 67:309-322.

[11]. Verma RK, Krishna DM, Garg S. Formulation aspects in the development of osmotically controlled oral drug delivery systems. J Control Release, 2002; 79:7-27.

[12]. Verma RK, Mishra B, Garg S. Osmotically controlled oral drug delivery. Drug Dev Ind Pharm 2000, 26:695-708.

[13]. Santus G., Baker RW. Osmotic drug delivery: a review of the patent literature. J Control Release, 1995; 33:1-21.

[14]. Davis DW. Method of swallowing a pill. US Patent 3418 999, December 31, 1968.

[15]. Stops F, Fell JT, Collett JH, Martini LG, Sharma HL, Smith AM. Citric acid prolongs the gastro-retention of a floating dosage form and increases bioavailability of riboflavin in the fasted state. Int J Pharm, 2006; 308:4-24.

[16]. Stops F, Fell JT, Collett JH, Martini LG. Floating dosage forms to prolong gastro-retention-The characterisation of calcium alginate beads. Int J Pharm, 2008; 350:301-311.

[17]. Hamdani J, Goole J, Moës AJ, Amighi K. In vitro and in vivo evaluation of floating riboflavin pellets developed using the melt pelletization process. Int J Pharm, 2006; 
323:86-92.

[18]. Ritschel WA. Targeting in the gastrointestinal tract: new approaches, Methods Find. Exp Clin Pharmacol, 1991; 13:313-336.

[19]. Ali J, Arora S, Ahuja A, Babbar AK, Sharma RK, Khar RK, Baboota S. Formulation and development of hydrodynamically balanced system for metformin: In vitro and in vivo evaluation. Eur J Pharm \& Biopharm, 2007; 67:196-201.

[20]. Xu X, Sun M, Zhi F, Hu Y. Floating matrix dosage form for phenoporlamine hydrochloride based on gas forming agent: In vitro and in vivo evaluation in healthy volunteers. Int J Pharm, 2006; 310:139-145.

[21]. Harrigan RM. Drug delivery device for preventing contact of undissolved drug with the stomach lining. US Patent 4055178 , October 25, 1977.

[22]. Singh BN, Kim KH. Floating drug delivery systems: an approach to oral controlled drug delivery via gastric retention. J Control Release, 2000; 63:235-259.

[23]. Chakrabarti S, Freedman JE. Dipyridamole, cerebrovascular disease, and the vasculature. Vascul Pharmacol. 2008; 48:143-9.

[24]. Kong SY, Lin R, Chen GM. Preparation of Dipyridamole Floating Tablets in Stomach. Chin J Pharm, 2003; 34:395-397.

[25]. Chavanpatil M, Jain P, Chaudhari S, Shear R, Vavia P. Development of sustained release gastroretentive drug delivery system for ofloxacin: In vitro and in vivo evaluation. Int $\mathrm{J}$ Phartm, 2005; 304:178-184.

[26]. Sato Y, Kawashima Y, Takeuchi H, Yamamoto $\mathrm{H}$. In vitro and in vivo evaluation of riboflavin-containing microballoons for a floating controlled drug delivery system in healthy humans. Int J Pharm, 2004; 275:97-107.

[27]. Joseph NJ, Lakshmi S, Jayakrishnan A. A floating-type oral dosage form for piroxicam based on hollow polycarbonate microspheres: in vitro and in vivo evaluation in rabbits. $\mathrm{J}$ Control Release, 2002; 79:71-79.

[28]. Schug BS, Brendel E, Wolf D, Wonnemann M, Blume HH. Formulation-dependent food effects demonstrated for nifedipine modified-release preparations marketed in the European Union. Eur J Pharm Sci, 2002; 15:279-285.

[29]. Shah VP, Tsong Y, Sathe P, Liu J. In Vitro Dissolution Profile Comparison-Statistics and Analysis of the Similarity Factor, f2. Pharm Res, 1998; 15:889-896.

[30]. Wang W, Cui G, Zhao Y, Liao J, Wang R, Zhou X. Studies on pharmacokinetics and relative bioavailability of dipyridamole sustained release pellets. Chin J New Drug, 2002; 11:612-615.

[31]. Qi Y, Gao Y, Zhang Y, Hao L, Fei H, Qu X, Zhu Y. The pharmacokinetics of dipyridamole administered in tragestrically in rats. Chin Pharm Bulletin, 2006; 22:379-380.

[32]. Lu EX, Jiang ZQ, Zhang QZ, Jiang XG. A water-insoluble drug monolithic osmotic tablet system utilizing gum Arabic as an osmotic, suspending expanding agent. J Control Release, 2003; 92:375-382.

[33]. Riegllman S, Collier P. The application of statistical moment theory to the evaluation of in vivo dissolution time and absorption. J Pharm. \& Biopharm, 1980; 8:509-534. 